\title{
Unusual Norrish Type I and Type II Nitro-Acyl Migration Transition State in the Photo-uncaging of 1-Acyl-7-nitroindolines Revealed by Computations.
}

Pierpaolo Morgante, Charitha Guruge, Yannick P. Ouedraogo, Nasri Nesnas*, Roberto Peverati*

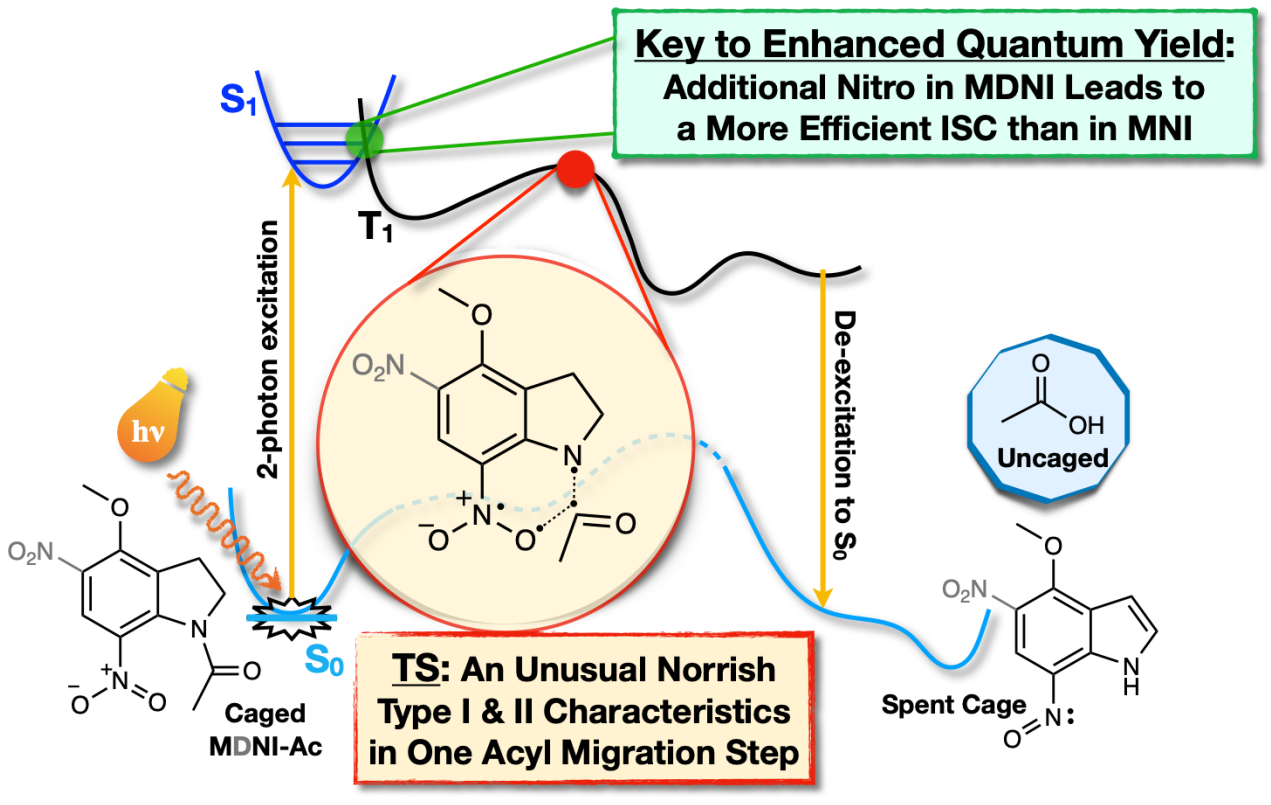

Abstract. The 7-nitroindolinyl family of caging chromophores has received a lot of attention in the past two decades. However, it is not clear if they undergo cyclization or migration upon photo-uncaging to release the active compound. In this study, we performed state-of-the-art density functional theory calculations to fully understand the photo-uncaging mechanism and we compared the probabilities of all plausible pathways. We found that the key transition state in the lowest-energy pathway involves an acyl migration. It possesses the characteristics of a combined Norrish Type I and a 1,6-nitro-acyl variation of a Norrish Type II mechanism, which has not been previously reported. We also introduced a new computational procedure that allows the estimation of intersystem crossing rate constants useful to compare the relative quantum yield of substituted cages. This procedure may pave the way for improved cage designs that possess higher quantum yields and more efficient agonist release. 
The ability to deliver or activate a compound to a specific site with precise timing is of extreme value. This is widely referred to as spatio-temporal control. ${ }^{1-4}$ Techniques that possess a spatio-temporal power have found numerous applications ranging from medicinal chemistry fields with photo-targeted therapeutics, ${ }^{1,3}$ such as cancer treatments, ${ }^{5-8}$ to the neuroscience where it becomes critical to activate selected neurons to study specific pathways ${ }^{1,9-12}$ and photo-initiated catalysis. $^{8}$ In the latter, emerging field of optogenetics, light sensitive channel rhodopsin receptors are genetically engineered in selected neurons rendering them light responsive., ${ }^{3,11,13-21}$ Additional methods of firing specific neurons employed a diverse array of photo-cleavable protecting groups attached to critically active sites of agonists. ${ }^{22-25}$ These photo-cleavable protecting group acquired the term photocages, or simply cages, as their attachment to an agonist necessarily incapacitates it, much like caging a ferocious animal.

Caged molecules are inert, and they need an external agent—such as a physical, chemical or mechanical force- ${ }^{8,26-29}$ to induce the release of the biologically active compound they are protecting. Light represents the most advantageous activator because it offers the ability to control the precise time and the location of the release. ${ }^{1-4}$ Although there are numerous classes of photocleavable groups, they share a common feature of absorbing light of specific wavelengths that leads to excitation from the ground state $\left(\mathrm{S}_{0}\right)$ to $\left(\mathrm{S}_{1,2,3 \ldots}\right)$ followed by an intersystem crossing (ISC) to the triplet state $\left(T_{1}\right){ }^{23,24,30-32}$ This leads to cleavage of the protecting group at the most labile bond which is critically positioned for such release (as shown in red for MDNI-Glu in Scheme $\mathbf{1}$ below). These mechanisms depend on the class of protecting groups employed, but some are not clearly understood, as mechanisms in excited states may not resemble the familiar mechanistic pathways commonly accepted for ground states. ${ }^{23,33}$

7-Nitroindolinyl cages have gained popularity at the turn of the century due to their optimal quantum yields and efficient release of agonists. ${ }^{34-36}$ Improvements of such cage systems led to the 
installation of a second nitro group at the 5 position that resulted in improved quantum yields..$^{37,38}$ Although the second nitro at the C-5 is not directly participating in the mechanism, as we will show later, its presence results in an improved quantum yield by at least 5 fold according to experimental data. ${ }^{39-41}$ The mechanisms of uncaging for the 7-indolinyl system was studied by at least three groups using kinetic, fast pulse IR, and computation..$^{37,42,43}$ However, the most recently reported computational mechanisms of uncaging do not explain the difference in quantum yield, which is an important parameter especially for consideration in future improved designs. We herein report a novel computational method that highlights the relative quantum yields of nitroindolinyl systems and also clarifies the mechanism with respect to the traditionally accepted formation of a cyclic intermediate.

The first mechanism for the light-initiated uncaging of MDNI-Glu (1) has been proposed by Ellis-Davies and coworkers in $2005,{ }^{38}$ and is based on kinetic data collected by Morrison et al. ${ }^{44}$ for another member of the 7-nitroindoline family. For simplicity, we will refer to this mechanism as the cyclization pathway (CP) from here on. According to their proposed mechanism, after irradiation, the reaction proceeds on the triplet surface via a cyclic intermediate (5). Subsequently, the system is deprotonated (3), and finally it delivers the free glutamate (7) to the reaction medium. According to the $\mathrm{CP}$ mechanism, the increased reactivity of MDNI is due to the influence of the nitro group in position 5 on the overall electronic structure of the indoline scaffold. ${ }^{38}$ A subsequent computational study by Pálfi et al. (the migration pathway, MP), ${ }^{43}$ however, did not report any cyclic intermediate, and attributed the increased reactivity of MDNI to a smaller difference in the enthalpy of activation of the rate-determining step $\left(\Delta \Delta \mathrm{H}_{\mathrm{RDS}}\right)$ of the uncaging reaction on the triplet surface. The most notable difference between the proposed CP mechanism and the MP mechanism is the formation of the cyclic intermediate 5 (see Scheme 1), and to which extent the substituents of the indoline scaffold affect each pathway. 


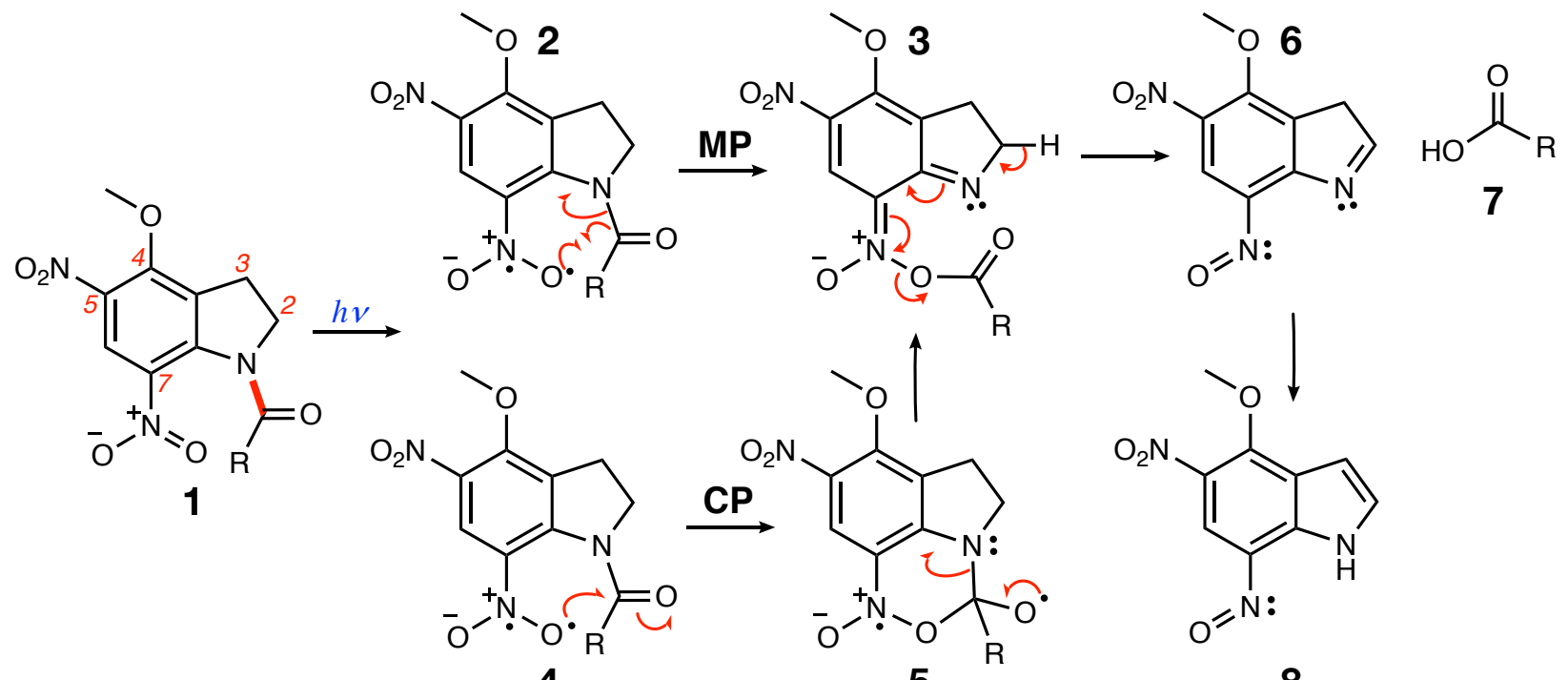

Scheme 1 Different proposed mechanisms for the uncaging of MDNI-Glu $\left(\mathrm{R}=\mathrm{CH}_{2} \mathrm{CH}_{2} \mathrm{CH}\left(\mathrm{NH}_{2}\right) \mathrm{COOH}\right)$ : Migration Pathway $(\mathrm{MP})$ - computational mechanism reported by Pálfi et al.;3 Cyclization Pathway (CP) - mechanism presumed by Ellis-Davies and coworkers, ${ }^{38}$ based on Morrison's experimental data. ${ }^{44}$

In this work, we aim to redeem the controversy by performing state-of-the-art density functional theory (DFT) calculations of the full reaction mechanism with the $\mathrm{M} 11^{45}$ exchangecorrelation functional approximation and the def2-TZVP basis set ${ }^{46}$ on top of geometries optimized at the B3LYP-D3(BJ)/def2-SVPD level of theory. ${ }^{46-52}$ We calculate and report also for the first time the Gibbs free energy profile-including both enthalpic and entropic corrections-of the reaction, in contrast to Pálfi et al. who reported only enthalpic contributions. Our results confirm that the cyclic intermediate does not form, but also show that the different substituents do not affect the mechanism of the reaction on the triplet surface as first reported by Pálfi et al. A novel computational protocol was therefore necessary to correlate the influence of the substituents with the sensitivity of the molecule towards light absorption. Our new protocol is based on the estimation of the rate constants of intersystem crossing (which has never been done previously for this class of compounds). Validation of the new protocol on external data shows promising results 
for its application for the prediction of quantum yields of molecules that have not been previously synthesized.

The reaction from MDNI-Ac (10) to 4-methoxy-5-nitro-7-nitrosoindole (8) and acetic acid is very favored from the thermodynamic point of view, having a calculated $\Delta G$ of $-38.7 \mathrm{kcal} / \mathrm{mol}$ (see Scheme 2 below). According to the CP mechanism, the cyclic intermediate 9 (Figure 1) is the key structure in the reaction mechanisms of MNI and MDNI, regardless of the leaving group. In fact, close inspection of its structure reveals that there are at least four bonds (labelled A, B, C or D in Figure 1) that can potentially break, leading to four different routes depending on which of these four bonds breaks first. We will label these routes according to the bond that breaks first.

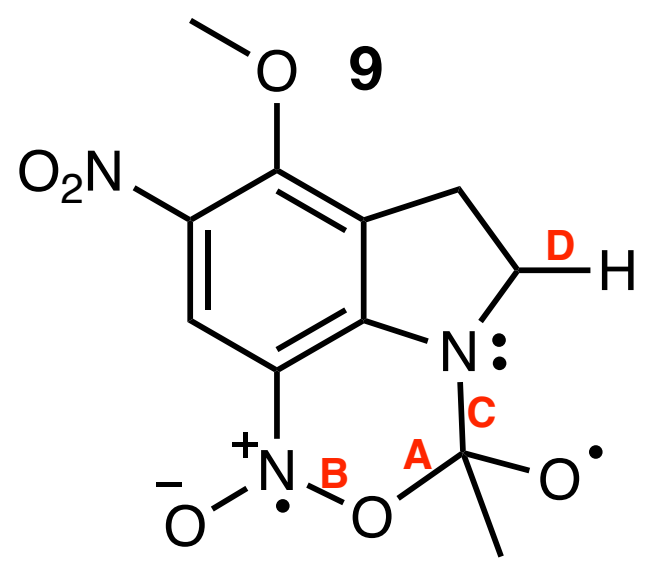

Figure 1 The cyclic intermediate for MDNI-Ac. This structure is similar to structure $\mathbf{5}$ in Scheme 1, but instead of glutamate it is bound to acetate.

Breaking the bond between carbon and oxygen (bond A in Figure 1) reverts the cyclic intermediate back to the reactant, and therefore does not lead to uncaging. Breaking bond B resulted in an unstable intermediate that reverted back to the cyclic structure itself during geometry optimization. For this reason, we did not explore this pathway (Cyclization Pathway-B, or CP-B) any further. The original CP mechanism involves breaking bond $\mathrm{C}$ first, followed by a concerted process, initiated by the deprotonation that leads to the reaction products, as seen in Scheme 1. Our 
calculations show that bond $\mathrm{C}$ can indeed be broken, but there is no deprotonation of the resulting intermediate. Instead, once the nitronic anhydride is formed, the leaving group departs, and then deprotonation occurs before the spent cage decays back to the singlet state (we termed this mechanism CP-C, see Scheme S1 in the Supplementary Information for more details). Last, we found the process starting from breaking bond $\mathrm{D}$, followed by the collapse of the cyclic intermediate is also possible, but energetically disfavored compared to the other pathways described above (breaking bond $\mathrm{D}$, which corresponds to an hydrogen atom abstraction, requires $21.6 \mathrm{kcal} / \mathrm{mol}$ for MDNI-Ac, see Scheme $\mathbf{S} 2$ in the Supplementary Information for the CP-D mechanism).

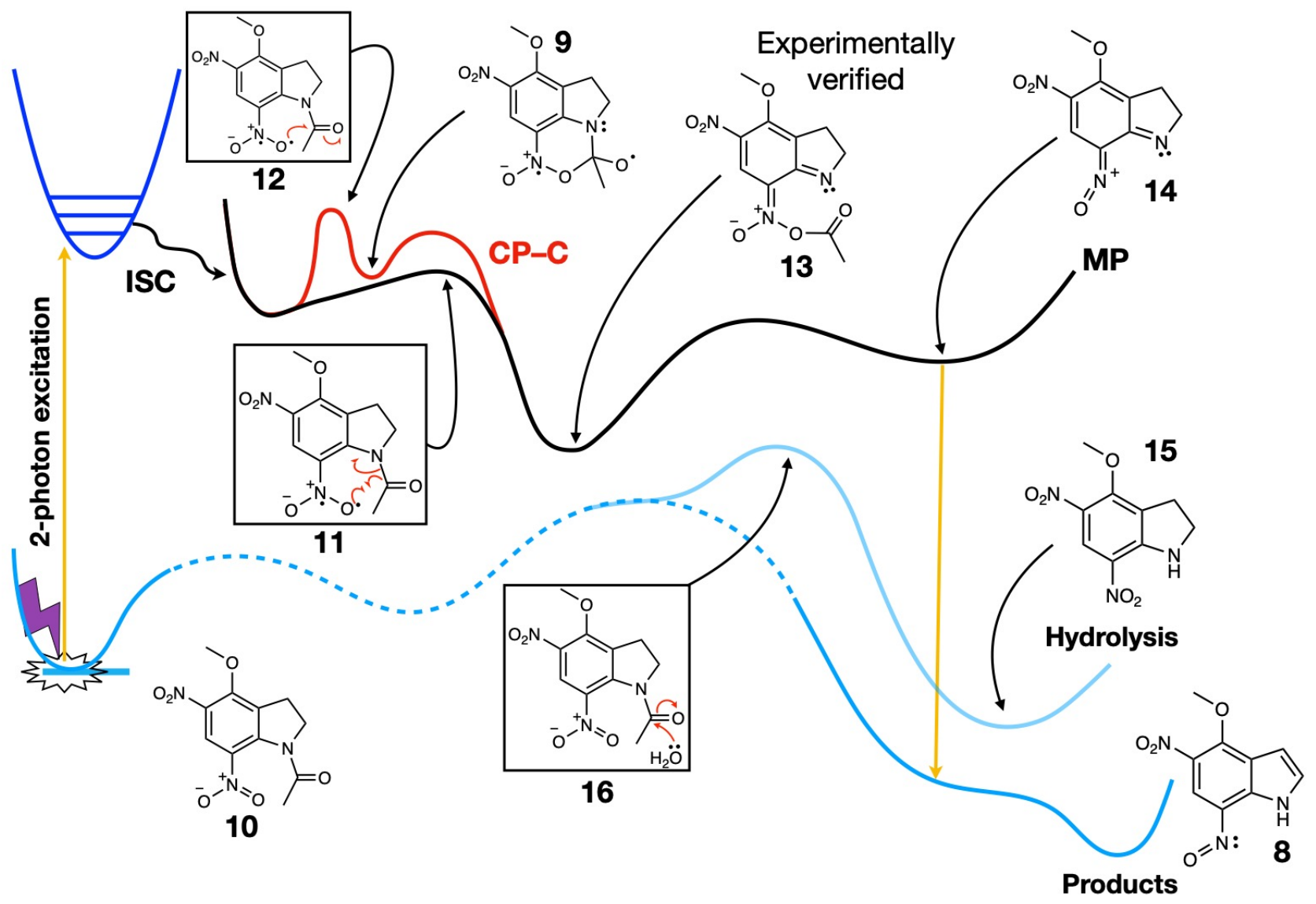

Scheme 2 Schematics of the main reaction pathways for MDNI-Ac: Migration Pathway (MP) mechanism of Pálfi et al., verified by our improved computational results; CP-C - Cyclization Pathway-C (CP-C) mechanism via cyclic intermediate (see text for detailed explanation of the pathways). The hydrolysis pathway on the singlet surface is also shown. The structures of the relevant transition states are reported in a box, while those of significant intermediates and products are reported without a box. 
The MP mechanism involves a migration process that occurs before the formation of the cyclic intermediate. According to our results, the energy required for the migration is lower than the energy required for cyclization, as seen in Scheme 2. This is consistent with the experimental observations, since it yields the same intermediate—-the nitronic anhydride—identified by Morrison et al. in their kinetic study, ${ }^{44}$ and by Cohen et al. in their fast IR spectroscopy study. ${ }^{42}$ Our computational results indicate that the MP is lower in energy than any of the CP ones discussed above, which should not be too surprising considering that the cyclic intermediate necessitates the deplanarization of the amide bond to place the available unoccupied orbital of the carbonyl in the accessible position for the oxygen radical to form the bond (Figure 2).

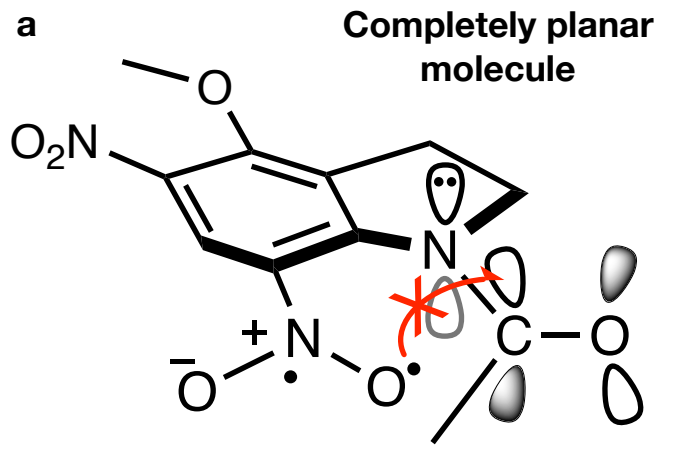

NOT IDEAL ANGLE FOR ATTACK

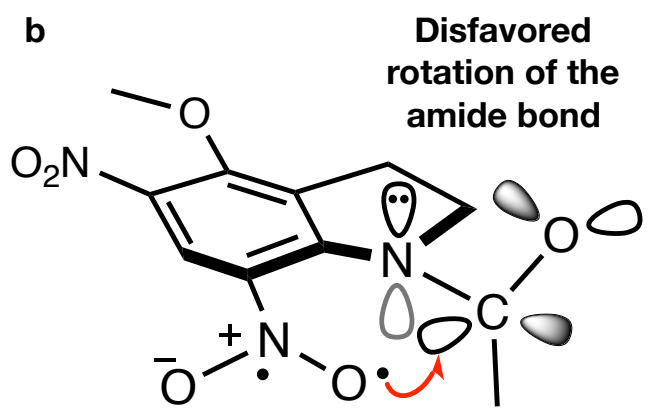

PREFERRED ANGLE FOR ATTACK

Figure 2 Key electronic effects for the cyclization pathway (CP). The oxygen radical in a is not positioned at the ideal Bürgi-Dunitz angle of $107^{\circ}$ relative to the plane of the molecule, and it is far away from the unoccupied orbital of the carbonyl group. The CP necessitates deplanarization of the amide bond via a rotation (b) in either sense, leading to two enantiomers (only one shown; see SI for more details). This leads to a higher energy pathway than the migration pathway (MP).

Furthermore, the migration of the acyl unit is facilitated by the inherent formation of the stabilized acyl radical, typically formed with classical Norrish ${ }^{53}$ type I alpha cleavages of excited carbonyl of ketones (Figure 3, part a). ${ }^{54-57}$ The 1,6-acyl migration effectively embodies 
characteristics of both type I and type II Norrish reactions in which the acyl undergoes an $\alpha$ cleavage as it transfers to the $\mathrm{O}$ radical of the nitro unit similar to the $\gamma-\mathrm{H}$ abstraction by triplet carbonyl in Norrish type II positioned at a 1,6-relationship (Figure 3, part b)..$^{53,57}$ In order to better understand this migration pathway, we computed the energy for an ethyl group migration (in place of the acetyl) and noted that it was higher in energy $(21.7 \mathrm{kcal} / \mathrm{mol}$, Figure 3 part c), despite being positioned in a 1,6-relationship to the nitro oxygen radical. This clearly reflects the stabilizing effect of an acyl radical in the transition state of the 1,6-migration. Therefore, based on the expected accuracy of our calculations $(\sim \pm 0.5 \mathrm{kcal} / \mathrm{mol}),{ }^{58-62}$ we can conclude that the reaction does not proceed through the cyclic structure (9) neither in the triplet nor in the singlet state.

a

Norrish Type I<smiles>[R]C([R])([R])C(C)=O</smiles>

Norrish Type II

b

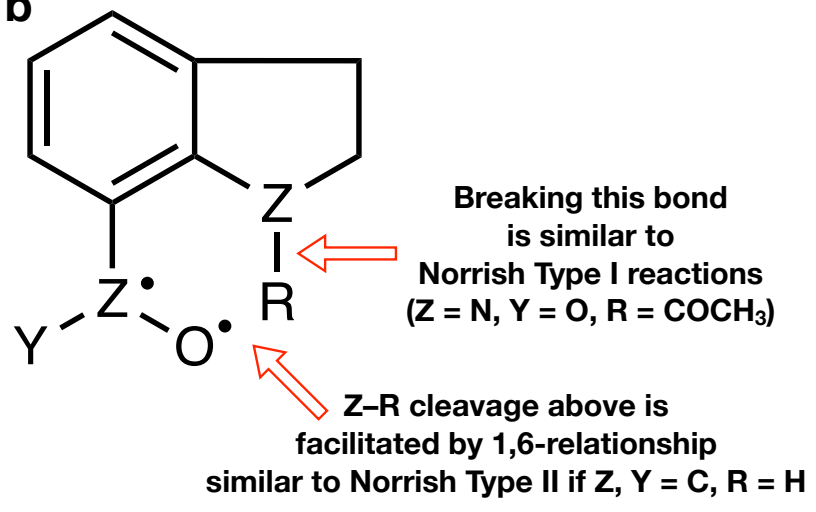

C

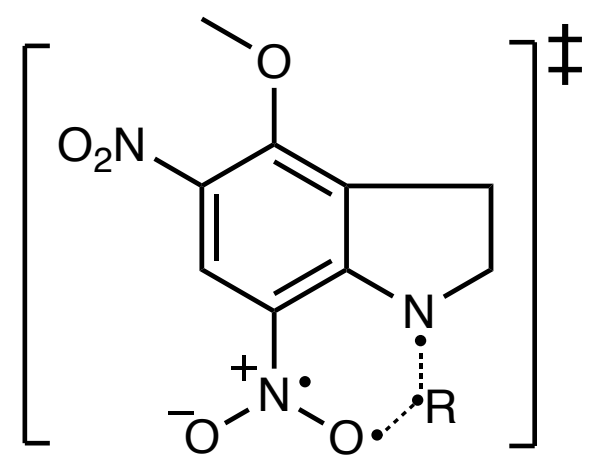

$R$ can be either $\mathrm{COCH}_{3}$ or $\mathrm{CH}_{2} \mathrm{CH}_{3}$ 
Figure 3 Schematics of the Norrish Type reactions (a). The MP pathway combines both Type I and Type II characteristics (b). Breaking the $\mathrm{Z}-\mathrm{R}$ bond $\left(\mathrm{Z}=\mathrm{N}, \mathrm{R}=\mathrm{COCH}_{3}\right)$ results in a Norrish Type I reaction, while the adjacent $\mathrm{Z}-\mathrm{O}$ bond is in a 1,6-relationship (Norrish Type II) and facilitates the cleavage of Z-R. Panel $\mathbf{c}$ shows the key migration transition state. If $\mathrm{R}=\mathrm{CH}_{2} \mathrm{CH}_{3}$, the reaction loses the Norrish Type I characteristic, and it is higher in energy by about $16 \mathrm{kcal} \mathrm{mol}^{-1}$.

Using the same computational strategy, we found that the modification of the substitution pattern on 7-nitroindoline is not as important as initially thought. Since the cyclic structure does not form, there is little influence of the substituents on the other steps of the reaction. In fact, the reported energy difference of $\Delta \Delta \mathrm{H}_{\mathrm{RDS}}=2.0 \mathrm{kcal} / \mathrm{mol}$ (calculated with B3LYP/6-31G*, ${ }^{47-52,63-65}$ which has an estimated error bar of $\sim \pm 1 \mathrm{kcal} / \mathrm{mol}$ ) between the MNI-Glu and MDNI-Glu pathways reported by Pálfi et al. reduces to only $\Delta \Delta G_{\mathrm{RDS}}=0.1 \mathrm{kcal} / \mathrm{mol}$ when the computational method is improved and the entropic corrections are appropriately included. This energy difference is too small to justify the experimentally observed difference in the quantum yields of the two systems. In addition, the results of Pálfi et al. show that the RDS of the reaction between MNI-Glu and MDNI-Glu is different than the one for $\alpha$-MNI-Glu and $\alpha$-MDNI-Glu. The only difference between these two classes of compounds is in the linking point of the cage, as also noted in ref. 41. In the first case, it is bound to the $\omega$-substituent of the glutamate chain, while in the second case the cage is bound to the carbon in the $\alpha$-position, hence the $\alpha-\mathrm{M}(\mathrm{D}) \mathrm{NI}$ notation. In ref. 43 the two $\alpha$ structures are called MNI-Ulg and MDNI-Ulg respectively. However, according to their reported data (see Table 1 in ref. 43), the enthalpy difference in the RDS for the $\alpha$-case is $\Delta \Delta H_{\mathrm{RDS}}=2.3$ $\mathrm{kcal} / \mathrm{mol}$, a value that is well within the accuracy of the method from the Glu case. For this reason, it is safe to assume that the effect of the substituents on the $\alpha$-mechanism will be similarly inconsequential, while the origin of the different quantum yields has to be searched somewhere else. Another major notable difference between the MP mechanism and our new results is that we did not find any reaction occurring on the singlet ground state. Pálfi et al. claim that hydrolysis and other 
side-reactions are problematic, as they can readily occur in the singlet ground state. ${ }^{43}$ This is in contradiction with both the experimental findings of Cohen et $\mathrm{al}^{42}$ and our exploration with the improved computational method. Our results show that the direct hydrolysis of MDNI-Ac has an activation energy of $43.0 \mathrm{kcal} / \mathrm{mol}$, which makes the reaction highly disfavored at room temperature (see Scheme S3 in the Supplementary Information). The other processes explored in ref. 43 that lead to the hydrolysis side-product have a barrier of at least $33.0 \mathrm{kcal} / \mathrm{mol}$. These barriers are high enough to make any side-reaction on the singlet surface negligible. It is noteworthy that if hydrolysis occurred, then the cage would lead to the uncontrolled release of the active compound, invalidating its application as caging agent as a whole, and would have been experimentally observed by Cohen et al. and in our laboratory. Last, we want to point out that the hydrolysis product (15) and the uncaging product (8) are different in that the former retains the nitro group while the photouncaging leads to the nitroso. However, both processes result in the release of acetic acid (from $\mathbf{1 0}$ and 13 respectively).

In summary, our improved results on the uncaging mechanism show that the lowest energy path proceeds as follow: After irradiation, the first step is the migration of the acetyl group from the nitrogen atom of indoline to one of the oxygen atoms of the neighboring nitro group. The corresponding transition structure (structure $\mathbf{1 1}$ in Scheme 3) is only $5.7 \mathrm{kcal} / \mathrm{mol}$ higher than the reactant, and it is fairly accessible at room temperature. As a comparison, the formation of the cyclic intermediate from the same reactant requires $12.6 \mathrm{kcal} / \mathrm{mol}$ (structure 12 in Scheme 2). In other words, the cyclization requires twice as much energy as the migration pathway, making it less favored despite having a non-prohibitive activation energy. After the first intermediate forms (structure 13), the subsequent step (18) has a barrier of $11.4 \mathrm{kcal} / \mathrm{mol}$, and thus it represents the ratedetermining step of the reaction. After the leaving group (acetate in this case) is released (18), a charged nitroso intermediate then forms (14), which readily deprotonates and then dis-excites, going 
back to the singlet surface as a spent cage (19). This is also consistent with the fact that molecules including an aromatic ring are more acidic in the excited state than in the ground state. ${ }^{66-69}$ Subsequently, the non-aromatic five-membered ring in $\mathbf{1 9}$ spontaneously tautomerizes to the fully aromatic indole system of 4-methoxy-5-nitro-7-nitrosoindole (8). The reaction mechanism for MDNI-Ac is outlined in Scheme 3.

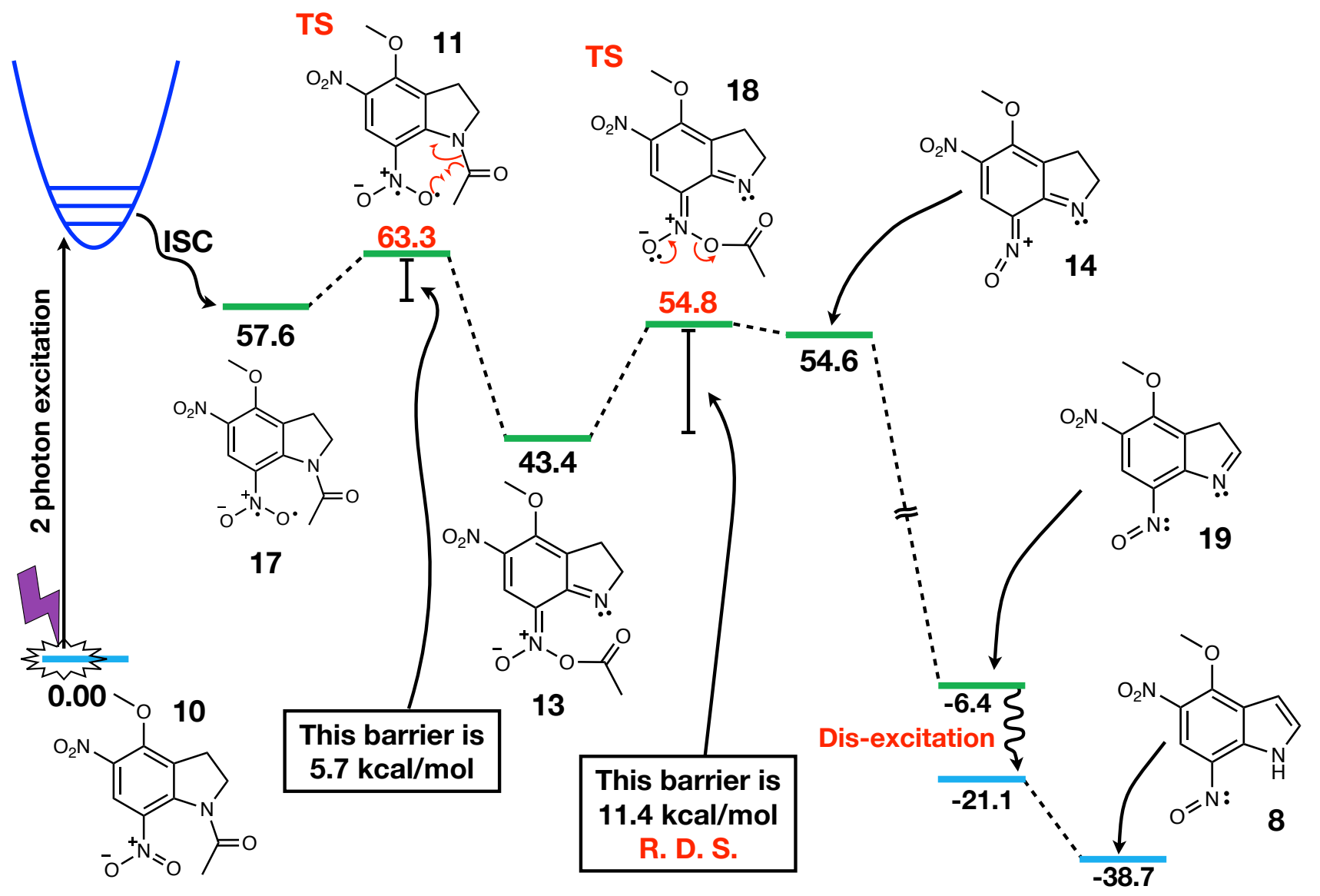

Scheme 3 The reaction mechanism for the uncaging of MDNI-Ac. The singlet surface is indicated in blue, while the triplet surface is indicated in green. All numerical values are in $\mathrm{kcal} / \mathrm{mol}$. All structures have been obtained at the B3LYP-D3(BJ)/def2-SVPD level of theory, ${ }^{46-52}$ while thermodynamic quantities have been computed at the M11/def2-TZVP level. ${ }^{45,46}$

Having excluded the uncaging mechanism on the triple surface as explanation of the quantum yield, the most probable steps that can affect the behavior of this family of molecules are the response towards the incident light and how readily the triplet state is populated. In fact, the true 
reactant is the molecule in the triplet state. We contend that controlling the amount of reactant in the triplet state is the key to achieve high quantum yields. In order to verify our claim, we performed time dependent density functional theory (TD-DFT) calculations using M11/def2-TZVP ${ }^{45,46}$ with B3LYP-D3(BJ)/def2-SVPD ${ }^{46-52}$ geometries in order to get access to the excited states of the molecules and therefore to the kinetic constant of intersystem crossing (vide infra). A singlet-triplet transition is strictly forbidden in non-relativistic quantum mechanics, but in practice, it can happen because of intersystem crossing (ISC). In general, a higher measured quantum yield for the entire process corresponds to a higher rate of ISC, as established for example for the borondipyrromethene (BODIPY) family of cages. ${ }^{25,70}$ However, there is no current methodology for the computational investigation of ISC for MNI and MDNI cages.

Borrowing from the work of Russo, Chiodo, Thiel and coworkers, ${ }^{71-73}$ we introduce here a novel computational strategy for the calculation of ISC of MNI and MDNI cages. As starting point, we estimate the rate constant for the ISC process, $k_{\mathrm{ISC}}$ as: $:^{74,75}$

$$
k_{\mathrm{ISC}}=\frac{4 \pi^{2}}{\mathrm{~h}} \mathrm{SOC}_{\mathrm{nm}}^{2} \frac{1}{\sqrt{4 \pi \lambda \mathrm{RT}}} \exp \left[-\frac{(\Delta \mathrm{E}+\lambda)^{2}}{4 \lambda \mathrm{RT}}\right]
$$

where $\lambda$ represents the vertical excitation energy between the singlet ground state and the $n$-th singlet excited state as calculated with TD-DFT, while $\Delta \mathrm{E}$ is the energy difference between the $n$-th singlet excited state and the $m$-th triplet state. $\mathrm{R}$ is the universal gas constant (equal to $8.314 \mathrm{~J} \mathrm{~mol}^{-1}$ $\mathrm{K}^{-1}$ ), $\mathrm{T}$ is $298.15 \mathrm{~K}$, and $\mathrm{h}$ is Planck's constant. $\mathrm{SOC}_{\mathrm{nm}}$ indicates the magnitude of spin-orbit coupling (SOC) between states $n$ and $m{ }^{76}$ We calculate the magnitude of SOC for both MNI-Ac and MDNI-Ac, more specifically between the first singlet excited state and the first triplet excited state for MNI-Ac and between the second singlet excited state and the third triplet excited state for MDNI-Ac. The numerical results are reported in Table 1. According to previous work, ${ }^{23,38,40}$ the quantum yield of MDNI-Glu is about five times the quantum yield of MNI-Glu. By comparing the 
two ISC rate constants we calculated, we can estimate the relative quantum yield of the reaction of MDNI-Ac to be 3.75 times that of MNI-Ac, in good agreement with the experimental data. As a further validation of our methodology, we calculated also the quantum yield of CDNI-Ac. The experimental quantum yield of CDNI is 4 to 5 times higher than the quantum yield of MNI, ${ }^{38,39,41}$ and it is about the same magnitude as the quantum yield of MDNI. We are pleased to notice that our calculated results are also in very good agreement with this experimental result.

Since our procedure is successful in predicting the ISC rate for MNI-Ac, MDNI-Ac, and CDNI-Ac, we extended it to include 5-chloro-4-methoxy-7-nitroindolinyl acetate (Cl-MNI-Ac) and 5-bromo-4-methoxy-7-nitroindolinyl acetate (Br-MNI-Ac), which have never been synthesized before. We expect the rate of ISC to be higher for Cl-MNI-Ac and Br-MNI-Ac due to the presence of an heavy atom ("heavy atom effect"), ${ }^{77-79}$ similarly to what happens for BODIPY. ${ }^{25}$ Our protocol predicts the chlorinated compound to be as reactive as MDNI, while the brominate compound to be more reactive than anything else (albeit by a thin margin), as we were expecting.

Table 1 Spin-orbit coupling (SOC) values, vertical excitation energies ( $\lambda$ ), singlet-triplet energy differences $(\Delta \mathrm{E})$, and Intersystem Crossing rate constants ( $\left.\mathrm{k}_{\mathrm{ISC}}\right)$ for selected molecules. Only the highest $\mathrm{k}_{\text {ISC }}$ is reported.

\begin{tabular}{|c|c|c|c|c|c|c|}
\hline Molecule & SOC $^{\mathbf{a}}$ & States $(\boldsymbol{n , m})$ & $\lambda^{\mathbf{b}}$ & $\Delta \mathbf{E}^{\mathbf{b}}$ & $\mathbf{k}_{\mathbf{I S C}}{ }^{\mathbf{c}}$ & $\begin{array}{c}\text { Relative } \\
\mathbf{Q u a n t u m}^{\mathbf{}} \\
\text { Yield }^{\mathbf{d}}\end{array}$ \\
\hline MNI & 115.9 & $\mathrm{~S}_{1}, \mathrm{~T}_{1}$ & 87.4 & -17.7 & $4.87 \times 10^{23}$ & 1.00 \\
\hline MDNI & 619.8 & $\mathrm{~S}_{2}, \mathrm{~T}_{3}$ & 88.4 & -15.3 & $1.83 \times 10^{24}$ & 3.75 \\
\hline CDNI & 585.7 & $\mathrm{~S}_{2}, \mathrm{~T}_{3}$ & 88.4 & -15.6 & $1.96 \times 10^{24}$ & 4.03 \\
\hline Cl-MNI-Ac & 124.8 & $\mathrm{~S}_{2}, \mathrm{~T}_{1}$ & 87.8 & -19.7 & $1.84 \times 10^{24}$ & 3.78 \\
\hline
\end{tabular}

\footnotetext{
${ }^{a}$ units are in $\mathrm{J} \mathrm{mol}^{-1} ;{ }^{\mathrm{b}}$ units are in $\mathrm{kcal} \mathrm{mol}^{-1} ;{ }^{\mathrm{c}}$ units are in $\mathrm{s}^{-1} \mathrm{~mol}^{-1} ;{ }^{\mathrm{d}}$ calculated with respect to MNI
}

In conclusion, we presented a detailed description of the reaction mechanism of 4-methoxy5,7-dinitroindolinyl acetate (MDNI-Ac) and 4-methoxy-7-nitroindolinyl acetate (MNI-Ac). We showed that the mechanism proceeds without formation of a cyclic intermediate in the triplet state, 
in contrast to previous beliefs. Instead, the reaction proceeds in the triplet state via an Norrish type I and II 1,6-acyl migration to the nitro group, which subsequently releases the leaving group of the nitronic ester, while the spent cage is recovered in the singlet ground state. The reason behind the success of MDNI as better caging group does not lie on lower activation energies, but rather on a better sensitivity towards light due to an increased rate of intersystem crossing, estimated using a procedure based on TD-DFT and spin-orbit coupling calculations, which we applied to 7nitroindolinyl-based cages for the first time. Our new computational protocol allowed for predictions about the reactivity of unexplored molecules, and guidance for their future synthesis.

\section{Methods.}

\section{Computational methods and models.}

All calculations were performed with the Gaussian $16^{80}$ program. For geometry optimizations, the B3LYP-D3(BJ) functional ${ }^{47-52}$ and the def2-SVPD basis set ${ }^{46,81-83}$ were used. The nature of all structures has been characterized through frequency analysis with the $\mathrm{M} 11^{45}$ functional and the def2-TZVP ${ }^{46}$ basis set. In all cases, a Lebedev integration grid of 99 radial and 590 angular points —abbreviated as $(99,590)$ - has been used. All calculations have been performed in water using the Conductor-Like Polarizable Continuum Model (C-PCM) ${ }^{84,85}$ framework. In addition, TDDFT calculations at the M11/def2-TZVP level have been performed. We used the pySOC program, ${ }^{72,73}$ which is based on TD-DFT output files obtained with Gaussian, to calculate the spinorbit coupling terms needed to estimate the ISC rate constants. More details about the computational procedure and sample input files can be found in the Supplementary Information.

\section{References.}


(1) Boyden, E. S.; Zhang, F.; Bamberg, E.; Nagel, G.; Deisseroth, K. Millisecond-Timescale, Genetically Targeted Optical Control of Neural Activity. Nat. Neurosci. 2005, 8 (9), 1263 1268.

(2) Spatiotemporal Mechanisms of Life. Nat. Chem. Biol. 2007, 3 (10), 593-593.

(3) Wang, K.; Liu, Y.; Li, Y.; Guo, Y.; Song, P.; Zhang, X.; Zeng, S.; Wang, Z. Precise Spatiotemporal Control of Optogenetic Activation Using an Acousto-Optic Device. PLoS ONE 2011, 6 (12), e28468.

(4) Zhu, P.; Fajardo, O.; Shum, J.; Zhang Schärer, Y.-P.; Friedrich, R. W. High-Resolution Optical Control of Spatiotemporal Neuronal Activity Patterns in Zebrafish Using a Digital Micromirror Device. Nat. Protoc. 2012, 7 (7), 1410-1425.

(5) Bach, T. M. H.; Takagi, H. Properties, Metabolisms, and Applications of L-Proline Analogues. Appl. Microbiol. Biotechnol. 2013, 97 (15), 6623-6634.

(6) Ashley, M. A.; Hirschi, J. S.; Izzo, J. A.; Vetticatt, M. J. Isotope Effects Reveal the Mechanism of Enamine Formation in L-Proline-Catalyzed $\alpha$-Amination of Aldehydes. J. Am. Chem. Soc. 2016, 138 (6), 1756-1759.

(7) Tanner, J. J.; Fendt, S.-M.; Becker, D. F. The Proline Cycle as a Potential Cancer Therapy Target. Biochemistry 2018, 57 (25), 3433-3444.

(8) Guruge, C.; Rfaish, S. Y.; Byrd, C.; Yang, S.; Starrett, A. K.; Guisbert, E.; Nesnas, N. Caged Proline in Photoinitiated Organocatalysis. J. Org. Chem. 2019, 84 (9), 5236-5244.

(9) Zemelman, B. V.; Lee, G. A.; Ng, M.; Miesenböck, G. Selective Photostimulation of Genetically ChARGed Neurons. Neuron 2002, 33 (1), 15-22.

(10) Zemelman, B. V.; Nesnas, N.; Lee, G. A.; Miesenbock, G. Photochemical Gating of Heterologous Ion Channels: Remote Control over Genetically Designated Populations of Neurons. Proc. Nat. Acad. Sci. 2003, 100 (3), 1352-1357.

(11) Miesenbock, G. The Optogenetic Catechism. Science 2009, 326 (5951), 395-399.

(12) Klapoetke, N. C.; Murata, Y.; Kim, S. S.; Pulver, S. R.; Birdsey-Benson, A.; Cho, Y. K.; Morimoto, T. K.; Chuong, A. S.; Carpenter, E. J.; Tian, Z.; Wang, J.; Xie, Y.; Yan, Z.; Zhang, Y.; Chow, B. Y.; Surek, B.; Melkonian, M.; Jayaraman, V.; Constantine-Paton, M.; Wong, G. K.-S.; Boyden, E. S. Independent Optical Excitation of Distinct Neural Populations. Nat. Methods 2014, 11 (3), 338-346.

(13) Nagel, G. Channelrhodopsin-1: A Light-Gated Proton Channel in Green Algae. Science 2002, 296 (5577), 2395-2398.

(14) Zhang, F.; Wang, L.-P.; Boyden, E. S.; Deisseroth, K. Channelrhodopsin-2 and Optical Control of Excitable Cells. Nat. Methods 2006, 3 (10), 785-792.

(15) Method of the Year 2010. Nat. Methods 2011, 8 (1), 1-1.

(16) Deisseroth, K. Optogenetics. Nat. Methods 2011, 8 (1), 26-29.

(17) Fenno, L.; Yizhar, O.; Deisseroth, K. The Development and Application of Optogenetics. Annu. Rev. Neurosci. 2011, 34 (1), 389-412.

(18) Pastrana, E. Optogenetics: Controlling Cell Function with Light. Nat. Methods 2011, 8 (1), 24 25.

(19) Aston-Jones, G.; Deisseroth, K. Recent Advances in Optogenetics and Pharmacogenetics. Brain Research 2013, 1511, 1-5.

(20) Mudiayi, D.; Wong, S.; Gruber, A. Optogenetics. In International Encyclopedia of the Social \& Behavioral Sciences; Elsevier, 2015; pp 268-273.

(21) Ronzitti, E.; Emiliani, V.; Papagiakoumou, E. Methods for Three-Dimensional All-Optical Manipulation of Neural Circuits. Front. Cell. Neurosci. 2018, 12, 469.

(22) Pettit, D. L.; Wang, S. S.-H.; Gee, K. R.; Augustine, G. J. Chemical Two-Photon Uncaging: A Novel Approach to Mapping Glutamate Receptors. Neuron 1997, 19 (3), 465-471. 
(23) Klán, P.; Šolomek, T.; Bochet, C. G.; Blanc, A.; Givens, R.; Rubina, M.; Popik, V.; Kostikov, A.; Wirz, J. Photoremovable Protecting Groups in Chemistry and Biology: Reaction Mechanisms and Efficacy. Chem. Rev. 2013, 113 (1), 119-191.

(24) Šolomek, T.; Wirz, J.; Klán, P. Searching for Improved Photoreleasing Abilities of Organic Molecules. Acc. Chem. Res. 2015, 48 (12), 3064-3072.

(25) Slanina, T.; Shrestha, P.; Palao, E.; Kand, D.; Peterson, J. A.; Dutton, A. S.; Rubinstein, N.; Weinstain, R.; Winter, A. H.; Klán, P. In Search of the Perfect Photocage: StructureReactivity Relationships in Meso-Methyl BODIPY Photoremovable Protecting Groups. J. Am. Chem. Soc. 2017, 139 (42), 15168-15175.

(26) Nagaoka, K.; Eboshi, T.; Takeishi, Y.; Tasaki, R.; Honda, K.; Imamura, K.; Sato, K. CarbonFree $\mathrm{H}_{2}$ Production from Ammonia Triggered at Room Temperature with an Acidic $\mathrm{RuO}_{2} / \gamma$ $\mathrm{Al}_{2} \mathrm{O}_{3}$ Catalyst. Sci. Adv. 2017, 3 (4), e1602747.

(27) Pizzolato, S. F.; Štacko, P.; Kistemaker, J. C. M.; van Leeuwen, T.; Otten, E.; Feringa, B. L. Central-to-Helical-to-Axial-to-Central Transfer of Chirality with a Photoresponsive Catalyst. J. Am. Chem. Soc. 2018, 140 (49), 17278-17289.

(28) Blanco, V.; Leigh, D. A.; Marcos, V. Artificial Switchable Catalysts. Chem. Soc. Rev. 2015, 44 (15), 5341-5370.

(29) Hölzl-Hobmeier, A.; Bauer, A.; Silva, A. V.; Huber, S. M.; Bannwarth, C.; Bach, T. Catalytic Deracemization of Chiral Allenes by Sensitized Excitation with Visible Light. Nature 2018, 564 (7735), 240-243.

(30) Maier, W.; Corrie, J. E. T.; Papageorgiou, G.; Laube, B.; Grewer, C. Comparative Analysis of Inhibitory Effects of Caged Ligands for the NMDA Receptor. J. Neurosci. Methods 2005, 142 (1), 1-9.

(31) Corrie, J. E. T.; Furuta, T.; Givens, R.; Yousef, A. L.; Goeldner, M. Photoremovable Protecting Groups Used for the Caging of Biomolecules. In Dynamic Studies in Biology; Goeldner, M., Givens, R. S., Eds.; Wiley-VCH Verlag GmbH \& Co. KGaA: Weinheim, FRG, 2005; pp 1-94.

(32) Kohl-Landgraf, J.; Buhr, F.; Lefrancois, D.; Mewes, J.-M.; Schwalbe, H.; Dreuw, A.; Wachtveitl, J. Mechanism of the Photoinduced Uncaging Reaction of Puromycin Protected by a 6-Nitroveratryloxycarbonyl Group. J. Am. Chem. Soc. 2014, 136 (9), 3430-3438.

(33) Pelliccioli, A. P.; Wirz, J. Photoremovable Protecting Groups: Reaction Mechanisms and Applications. Photochem. Photobiol. Sci. 2002, 1 (7), 441-458.

(34) Papageorgiou, G.; Ogden, D. C.; Barth, A.; Corrie, J. E. T. Photorelease of Carboxylic Acids from 1-Acyl-7-Nitroindolines in Aqueous Solution: Rapid and Efficient Photorelease of LGlutamate'. J. Am. Chem. Soc. 1999, 121 (27), 6503-6504.

(35) Papageorgiou, G.; Corrie, J. E. T. Effects of Aromatic Substituents on the Photocleavage of 1-Acyl-7-Nitroindolines. Tetrahedron 2000, 56 (41), 8197-8205.

(36) Matsuzaki, M.; Ellis-Davies, G. C. R.; Nemoto, T.; Miyashita, Y.; Iino, M.; Kasai, H. Dendritic Spine Geometry Is Critical for AMPA Receptor Expression in Hippocampal CA1 Pyramidal Neurons. Nat. Neurosci 2001, 4 (11), 1086-1092.

(37) Papageorgiou, G.; Ogden, D.; Kelly, G.; Corrie, J. E. T. Synthetic and Photochemical Studies of Substituted 1-Acyl-7-Nitroindolines. Photochem. Photobiol. Sci. 2005, 4 (11), 887.

(38) Fedoryak, O. D.; Sul, J.-Y.; Haydon, P. G.; Ellis-Davies, G. C. R. Synthesis of a Caged Glutamate for Efficient One- and Two-Photon Photorelease on Living Cells. Chem. Commun. 2005, No. 29, 3664.

(39) Ellis-Davies, G. C. R.; Matsuzaki, M.; Paukert, M.; Kasai, H.; Bergles, D. E. 4Carboxymethoxy-5,7-Dinitroindolinyl-Glu: An Improved Caged Glutamate for Expeditious Ultraviolet and Two-Photon Photolysis in Brain Slices. J. Neurosci. 2007, 27 (25), 6601-6604. 
(40) Comitz, R. L.; Ouedraogo, Y. P.; Nesnas, N. Unambiguous Evaluation of the Relative Photolysis Rates of Nitro Indolinyl Protecting Groups Critical for Brain Network Studies. Analytical Chemistry Research 2015, 3, 20-25.

(41) Guruge, C.; Ouedraogo, Y. P.; Comitz, R. L.; Ma, J.; Losonczy, A.; Nesnas, N. Improved Synthesis of Caged Glutamate and Caging Each Functional Group. ACS Chem. Neurosci. 2018, 9 (11), 2713-2721.

(42) Cohen, A. D.; Helgen, C.; Bochet, C. G.; Toscano, J. P. The Mechanism of Photoinduced Acylation of Amines by N-Acyl-5,7-Dinitroindoline as Determined by Time-Resolved Infrared Spectroscopy. Org. Lett. 2005, 7 (14), 2845-2848.

(43) Pálfi, D.; Chiovini, B.; Szalay, G.; Kaszás, A.; Turi, G. F.; Katona, G.; Ábrányi-Balogh, P.; Szőri, M.; Potor, A.; Frigyesi, O.; Lukácsné Haveland, C.; Szadai, Z.; Madarász, M.; VasanitsZsigrai, A.; Molnár-Perl, I.; Viskolcz, B.; Csizmadia, I. G.; Mucsi, Z.; Rózsa, B. High Efficiency Two-Photon Uncaging Coupled by the Correction of Spontaneous Hydrolysis. Org. Biomol. Chem. 2018, 16 (11), 1958-1970.

(44) Morrison, J.; Wan, P.; Corrie, J. E. T.; Papageorgiou, G. Mechanisms of Photorelease of Carboxylic Acids from 1-Acyl-7-Nitroindolines in Solutions of Varying Water Content. Photochem. Photobiol. Sci. 2002, 1 (12), 960.

(45) Peverati, R.; Truhlar, D. G. Improving the Accuracy of Hybrid Meta-GGA Density Functionals by Range Separation. J. Phys. Chem. Lett. 2011, 2, 2810-2817.

(46) Weigend, F.; Ahlrichs, R. Balanced Basis Sets of Split Valence, Triple Zeta Valence and Quadruple Zeta Valence Quality for H to Rn: Design and Assessment of Accuracy. Phys. Chem. Chem. Phys. 2005, 7 (18), 3297-3305.

(47) Vosko, S. H.; Wilk, L.; Nusair, M. Accurate Spin-Dependent Electron Liquid Correlation Energies for Local Spin-Density Calculations: A Critical Analysis. Can. J. Phys. 1980, 58 (8), 1200-1211.

(48) Becke, A. D. Density-Functional Exchange-Energy Approximation with Correct AsymptoticBehavior. Phys. Rev. A 1988, 38 (6), 3098-3100.

(49) Lee, C.; Yang, W.; Parr, R. G. Development of the Colle-Salvetti Correlation-Energy Formula Into a Functional of the Electron-Density. Phys. Rev. B 1988, 37, 785-789.

(50) Becke, A. D. Density-Functional Thermochemistry. III. The Role of Exact Exchange. J. Chem. Phys. 1993, 98 (7), 5648-5652.

(51) Stephens, P. J.; Devlin, F. J.; Chabalowski, C. F.; Frisch, M. J. Ab Initio Calculation of Vibrational Absorption and Circular Dichroism Spectra Using Density Functional Force Fields. J. Phys. Chem. 1994, 98 (45), 11623-11627.

(52) Grimme, S.; Ehrlich, S.; Goerigk, L. Effect of the Damping Function in Dispersion Corrected Density Functional Theory. J. Comput. Chem. 2011, 32 (7), 1456-1465.

(53) Norrish, R. G. W.; Bamford, C. H. Photo-Decomposition of Aldehydes and Ketones. Nature 1937, 140 (3535), 195-196.

(54) Yang, N.-Chu.; Feit, E. D.; Hui, M. Him.; Turro, N. J.; Dalton, J. Christopher. Photochemistry of Di-Tert-Butyl Ketone and Structural Effects on the Rate and Efficiency of Intersystem Crossing of Aliphatic Ketones. J. Am. Chem. Soc. 1970, 92 (23), 6974-6976.

(55) Heine, H. G.; Hartmann, W.; Kory, D. R.; Magyer, J. C.; Hoyle, C. E.; McVey, J. K.; Lewis, F. D. Photochemical .Alpha. Cleavage and Free-Radical Reactions of Some Deoxybenzoins. J. Org. Chem. 1974, 39 (5), 691-698.

(56) Lewis, F. D.; Hoyle, C. H.; Magyar, J. G.; Heine, H. G.; Hartmann, W. Photochemical .Alpha. Cleavage of Ketones in Solution. VI. Substituent Effects on the Photochemical .Alpha. Cleavage of Deoxybenzoin. J. Org. Chem. 1975, 40 (4), 488-492. 
(57) Paulson, S. E.; Liu, D.-L.; Orzechowska, G. E.; Campos, L. M.; Houk, K. N. Photolysis of Heptanal. J. Org. Chem. 2006, 71 (17), 6403-6408.

(58) Mardirossian, N.; Head-Gordon, M. Thirty Years of Density Functional Theory in Computational Chemistry: An Overview and Extensive Assessment of 200 Density Functionals. Mol. Phys. 2017, 115 (19), 2315-2372.

(59) Goerigk, L.; Hansen, A.; Bauer, C.; Ehrlich, S.; Najibi, A.; Grimme, S. A Look at the Density Functional Theory Zoo with the Advanced GMTKN55 Database for General Main Group Thermochemistry, Kinetics and Noncovalent Interactions. Phys. Chem. Chem. Phys. 2017, 19 (48), 32184-32215.

(60) Yu, H. S.; He, X.; Li, S. L.; Truhlar, D. G. MN15: A Kohn-Sham Global-Hybrid ExchangeCorrelation Density Functional with Broad Accuracy for Multi-Reference and SingleReference Systems and Noncovalent Interactions. Chem. Sci. 2016, 7 (8), 5032-5051.

(61) Morgante, P.; Peverati, R. Statistically Representative Databases for Density Functional Theory Via Data Science. Phys. Chem. Chem. Phys. 2019, 21 (35), 19092-19103.

(62) Migliore, A. How To Extract Quantitative Information on Electronic Transitions from the Density Functional Theory "Black Box." J. Chem. Theory Comput. 2019, 15 (9), 4915-4923.

(63) Ditchfield, R.; Hehre, W. J.; Pople, J. A. Self-Consistent Molecular-Orbital Methods. IX. An Extended Gaussian-Type Basis for Molecular-Orbital Studies of Organic Molecules. J. Chem. Phys. 1971, 54 (2), 724-728.

(64) Hehre, W. J.; Ditchfield, R.; Pople, J. A. Self_Consistent Molecular Orbital Methods. XII. Further Extensions of Gaussian-Type Basis Sets for Use in Molecular Orbital Studies of Organic Molecules. J. Chem. Phys. 1972, 56 (5), 2257-2261.

(65) Hariharan, P. C.; Pople, J. A. The Influence of Polarization Functions on Molecular Orbital Hydrogenation Energies. Theor. Chim. Acta 1973, 28 (3), 213-222.

(66) Rosenberg, J. L.; Brinn, I. Excited State Dissociation Rate Constants in Naphthols. J. Phys. Chem. 1972, 76 (24), 3558-3562.

(67) Arnaut, L. G.; Formosinho, S. J. Excited-State Proton Transfer Reactions I. Fundamentals and Intermolecular Reactions. J. Photochem. Photobiol. A: Chemistry 1993, 75 (1), 1-20.

(68) Tolbert, L. M.; Solntsev, K. M. Excited-State Proton Transfer: From Constrained Systems to "Super" Photoacids to Superfast Proton Transfer ${ }^{\dagger}$. Acc. Chem. Res. 2002, 35 (1), 19-27.

(69) Agmon, N.; Bakker, H. J.; Campen, R. K.; Henchman, R. H.; Pohl, P.; Roke, S.; Thämer, M.; Hassanali, A. Protons and Hydroxide Ions in Aqueous Systems. Chem. Rev. 2016, 116 (13), 7642-7672.

(70) Buck, A. T.; Beck, C. L.; Winter, A. H. Inverted Substrate Preferences for Photochemical Heterolysis Arise from Conical Intersection Control. J. Am. Chem. Soc. 2014, 136 (25), 89338940.

(71) Chiodo, S.; Russo, N. Determination of Spin-Orbit Coupling Contributions in the Framework of Density Functional Theory. J. Comput. Chem. 2008, 29 (6), 912-920.

(72) Chiodo, S. G.; Leopoldini, M. MolSOC: A Spin-Orbit Coupling Code. Computer Physics Communications 2014, 185 (2), 676-683.

(73) Gao, X.; Bai, S.; Fazzi, D.; Niehaus, T.; Barbatti, M.; Thiel, W. Evaluation of Spin-Orbit Couplings with Linear-Response Time-Dependent Density Functional Methods. J. Chem. Theory Comput. 2017, 13 (2), 515-524.

(74) Balzani, V.; Juris, A.; Venturi, M.; Campagna, S.; Serroni, S. Luminescent and Redox-Active Polynuclear Transition Metal Complexes ${ }^{\dagger}$. Chem. Rev. 1996, 96 (2), 759-834.

(75) Orozco-Gonzalez, Y.; Coutinho, K.; Peon, J.; Canuto, S. Theoretical Study of the Absorption and Nonradiative Deactivation of 1-Nitronaphthalene in the Low-Lying Singlet and Triplet 
Excited States Including Methanol and Ethanol Solvent Effects. J. Chem. Phys. 2012, 137 (5), 054307.

(76) Marian, C. M. Spin-Orbit Coupling and Intersystem Crossing in Molecules: Spin-Orbit Coupling. WIREs Comput Mol Sci 2012, 2 (2), 187-203.

(77) Giachino, G. G.; Kearns, D. R. Nature of the External Heavy-Atom Effect on Radiative and Nonradiative Singlet-Triplet Transitions. J. Chem. Phys. 1970, 52 (6), 2964-2974.

(78) Koziar, J. C.; Cowan, D. O. Photochemical Heavy-Atom Effects. Acc. Chem. Res. 1978, 11 (9), 334-341.

(79) Saigusa, H.; Azumi, T. Internal Heavy Atom Effect on the Triplet Spin Sublevels of the Lowest Triplet State of Naphthalene. I. Radiative and Nonradiative Decays of the Spin Sublevels of 1-halonaphthalenes. The Journal of Chemical Physics 1979, 71 (3), 1408-1413.

(80) Frisch, M. J.; Trucks, G. W.; Schlegel, H. B.; Scuseria, G. E.; Robb, M. A.; Cheeseman, J. R.; Scalmani, G.; Barone, V.; Petersson, G. A.; Nakatsuji, H.; Li, X.; Caricato, M.; Marenich, A. V.; Bloino, J.; Janesko, B. G.; Gomperts, R.; Mennucci, B.; Hratchian, H. P.; Ortiz, J. V.; Izmaylov, A. F.; Sonnenberg, J. L.; Williams-Young, D.; Ding, F.; Lipparini, F.; Egidi, F.; Goings, J.; Peng, B.; Petrone, A.; Henderson, T.; Ranasinghe, D.; Zakrzewski, V. G.; Gao, J.; Rega, N.; Zheng, G.; Liang, W.; Hada, M.; Ehara, M.; Toyota, K.; Fukuda, R.; Hasegawa, J.; Ishida, M.; Nakajima, T.; Honda, Y.; Kitao, O.; Nakai, H.; Vreven, T.; Throssell, K.; Montgomery, J. A., Jr.; Peralta, J. E.; Ogliaro, F.; Bearpark, M. J.; Heyd, J. J.; Brothers, E. N.; Kudin, K. N.; Staroverov, V. N.; Keith, T. A.; Kobayashi, R.; Normand, J.; Raghavachari, K.; Rendell, A. P.; Burant, J. C.; Iyengar, S. S.; Tomasi, J.; Cossi, M.; Millam, J. M.; Klene, M.; Adamo, C.; Cammi, R.; Ochterski, J. W.; Martin, R. L.; Morokuma, K.; Farkas, O.; Foresman, J. B.; Fox, D. J. Gaussian 16 Revision A.03; 2016.

(81) Feller, D. The Role of Databases in Support of Computational Chemistry Calculations. J. Comput. Chem. 1996, 17 (13), 1571-1586.

(82) Schuchardt, K. L.; Didier, B. T.; Elsethagen, T.; Sun, L.; Gurumoorthi, V.; Chase, J.; Li, J.; Windus, T. L. Basis Set Exchange: A Community Database for Computational Sciences. J. Chem. Inf. Model. 2007, 47 (3), 1045-1052.

(83) Pritchard, B. P.; Altarawy, D.; Didier, B.; Gibson, T. D.; Windus, T. L. New Basis Set Exchange: An Open, Up-to-Date Resource for the Molecular Sciences Community. J. Chem. Inf. Model. 2019, 59 (11), 4814-4820.

(84) Barone, V.; Cossi, M. Quantum Calculation of Molecular Energies and Energy Gradients in Solution by a Conductor Solvent Model. J. Phys. Chem. A 1998, 102 (11), 1995-2001.

(85) Cossi, M.; Rega, N.; Scalmani, G.; Barone, V. Energies, Structures, and Electronic Properties of Molecules in Solution with the C-PCM Solvation Model. J. Comput. Chem. 2003, 24 (6), 669-681.

\section{Acknowledgements.}

Part of these calculations have been performed on Florida Tech's BlueShark cluster, which was funded by the National Science Foundation under Grant No. CNS 
09-23050. The authors also wish to thank the National Institutes of Health for providing funds for this project and for supporting C.G. (R15-GM112119-01A1).

\section{Author Contributions.}

P.M. and R.P. conducted all computations. C.G. synthesized the caged compounds and analyzed their photo-physical properties. Y.P.O. performed initial DFT calculations that steered the team for further investigation. N.N. directed this project and through discussions of results with R.P. and P.M. came to the conclusion of an unusual Norrish I \& II combined TS. P.M., R.P., and N.N. prepared the manuscript with contributions from all authors.

\section{Competing Interests.}

The Authors declare no competing interests.

\section{Additional Information.}

Supplementary Information is available for this paper with details of the different mechanisms, sample Gaussian 16 input files, and all appropriate references for the computational methodologies. Cartesian coordinates for all the species reported are available in a separate folder (Geometries_Uncaging.zip). 